\title{
Prompt fission neutron emission in the reaction ${ }^{235} U(n, f)$
}

\author{
Alf Göök ${ }^{\star}$, Franz-Josef Hambsch, and Stephan Oberstedt \\ European Commission, Joint Research Centre, Directorate G - Nuclear Safety and Security, Unit G.2, \\ Retieseweg 111, 2440 Geel, Belgium
}

\begin{abstract}
Experimental activities at JRC-Geel on prompt fission neutron (PFN) emission in response to OECD/NEA nuclear data requests are presented in this contribution. Specifically, on-going investigations of PFN emission from the reaction ${ }^{235} U(n, f)$ in the region of the resolved resonances, taking place at the GELINA facility, are presented. The focus of this contribution lies on studies of PFN correlations with fission fragment properties. The experiment employs a scintillation detector array for neutron detection, while fission fragment properties are determined via the double kinetic energy technique using a position sensitive twin ionization chamber. This setup allows us to study several correlations between properties of neutron and fission fragments simultaneously. Results on PFN correlations with fission fragment properties from the present study differ significantly from earlier studies on this reaction, induced by thermal neutrons.
\end{abstract}

\section{Introduction}

Experimental investigations of prompt fission neutrons and fission fragment properties in resonance neutron induced fission on ${ }^{235} \mathrm{U}$ are taking place at the GELINA facility of the JRC-Geel. Improved knowledge of the properties of prompt fission neutrons (PFN), their multiplicities, as well as their energy and angular distributions are not only of interest for questions related to the neutron emission itself, but also to questions relevant to the formation of the fission fragments, the sharing of excitation energy among them and the time scale of the process. In recent years large efforts have been put into the modeling of PFN emission in fission [1]. The efforts are quite successfu in both consistency between the different approaches and reproducing available experimental data. However, in the case of ${ }^{235} \mathrm{U}(\mathrm{n}, \mathrm{f})$ difficulties have been encountered [2], especially regarding the dependency of $\bar{v}$ on the fragment total kinetic energy (TKE). In Ref. [3] possible deficiencies in the experimental data were pointed out. For these reasons it is interesting to revisit PFN and fragment correlations in ${ }^{235} \mathrm{U}(\mathrm{n}, \mathrm{f})$ experimentally.

\section{Experimental setup}

The experiment has been performed at the GELINA facility of the JRC-Geel site. The experimental setup, illustrated in Fig. 1, is located at a distance of $9.2 \mathrm{~m}$ from the GELINA neutron source. Fission fragments are detected in a position sensitive twin ionization chamber (IC). This detector has been

^e-mail: alf.gook@ec.europa.eu 


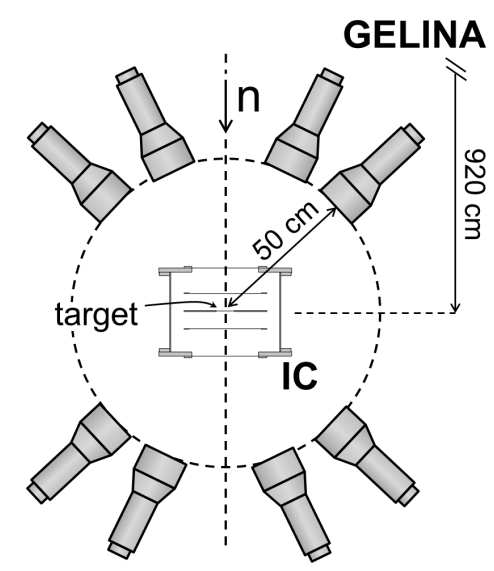

Figure 1. Schematic representation of the experimental setup located at the GELINA facility. The ${ }^{235} \mathrm{U}$-target is placed inside the twin ionization chamber (IC), $9.2 \mathrm{~m}$ away from the neutron producing target. The neutron detectors are placed in a quasi spherical geometry around the IC at a distance of about $50 \mathrm{~cm}$ from the center of the ${ }^{235} \mathrm{U}$-target.

developed at JRC-Geel, and is described in Ref. [4] in detail. Essentially, it is a standard twin Frisch grid ionization chamber with the anode plates replaced by position sensitive readouts. It is capable of determining the fission axis orientation in space with a resolution of $7^{\circ}$, simultaneously with the fission fragment masses and energies.

Neutrons are detected in an array of proton recoil scintillators. The array consist of 19 NE213 equivalent BC-501 liquid scintillators, 3 p-terphenyl and 1 stilbene crystal scintillators. The detectors are placed in a quasi-spherical geometry around the center of the ${ }^{235} \mathrm{U}$-target at distance of about $50 \mathrm{~cm}$. The actual position of the detectors relative to the IC has been determined with high precision using a ROMER $\operatorname{arm}^{1}$ measuring device.

The data acquisition is based on wave-form digitizers, sampling the detector signals at 400 MSam$\mathrm{ple} / \mathrm{s}$ with 14-bit resolution. A fission trigger derived from the charge induced on the central cathode of the IC, triggers the data acquisition. For every fission trigger digital wave-forms of all neutron detectors as well as the IC's electrodes are stored on disk, together with time-stamp information, for off-line analysis. The time stamp is reset by every beam pulse from the GELINA and used to determine the incident neutron time-of-flight (ToF).

\section{Data analysis}

\subsection{PFN data}

Events in the scintillation detectors corresponding to neutrons are selected by means of pulse-shape discrimination (PSD). The PFN energy is measured by means of the ToF technique, with a resolution of $\sim 1 \mathrm{~ns}$ (FWHM). In addition to PFNs, the fission is also accompanied by prompt fission $\gamma$-rays, which might be detected in the scintillation detectors as well. Most of the $\gamma$-emission takes place within a few ns after the instant of fission. Therefore, the range of high-energy neutrons is most sensitive to false events induced by $\gamma$-rays. The PSD is not able to give a clean discrimination between $\gamma$-rays and neutron events for pulse heights corresponding to a proton recoil energy smaller than $\sim 1 \mathrm{MeV}$. In order to reduce the background caused by the $\gamma$-emission a dynamic light-threshold [5] is applied.

Each detector in the SCINTIA array has been characterized using the PFNs from ${ }^{252} \mathrm{Cf}(\mathrm{sf})$, with experimental setup and procedures described in Ref. [6]. To correct for the energy dependent detection

\footnotetext{
${ }^{1}$ http://www.hexagonmi.com/products/portable-measuring-arms/romer-absolute-arm
} 
efficiency and multiple scattering of neutrons on the surrounding materials, the setup is modeled with GEANT4. The simulation uses experimentally determined proton light output functions, for other recoil-particle species literature data are used [7, 8]. The Monte-Carlo model has been validated against the standard PFN spectrum [9] from the spontaneous fission decay of ${ }^{252} \mathrm{Cf}$.
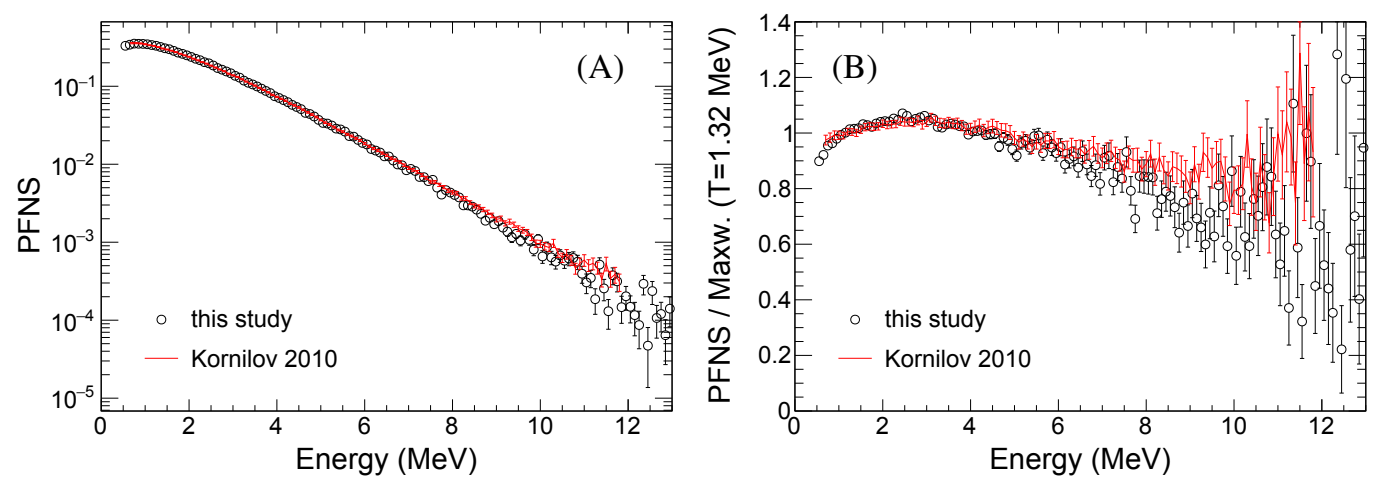

Figure 2. (A) The prompt fission neutron spectrum in the laboratory frame compared to data from cold-neutron induced fission from Kornilov et al. [10]. (B) The prompt fission neutron spectrum represented as a ratio to a Maxellian with a temperature of $1.32 \mathrm{MeV}$.

In Fig. 2 the ${ }^{235} \mathrm{U}(\mathrm{n}, \mathrm{f})$ PFN spectrum observed in this study, when selecting the incident neutron energy range $[0.3 \mathrm{eV}, 45 \mathrm{keV}]$, is compared to the spectrum from cold neutron induced fission determined by Kornilov et al. [10]. The two spectra are in quite close agreement, although the spectrum observed in the resonance region is slightly softer.

\subsection{Determination of fission fragment properties}

The fission fragment mass and kinetic energy before neutron emission is obtained by means of the well-established double kinetic energy (2E) technique, which relates the masses $m_{1,2}^{*}$ and energies $E_{1,2}^{*}$ before neutron emission in a binary fission event according to

$$
m_{1,2}^{*}=m_{c n} \cdot \frac{E_{2,1}^{*}}{E_{1}^{*}+E_{2}^{*}},
$$

where $m_{c n}$ is the mass of the compound nucleus undergoing fission. Under the assumption of isotropic neutron emission from fully accelerated fragments, the energies before neutron emission $E^{*}$ are related to the energies after neutron emission $E$ according to

$$
E^{*}=E \cdot \frac{m^{*}}{m^{*}-\bar{v}\left(m^{*}, \mathrm{TKE}\right)},
$$

where $\bar{v}$ is the number of neutrons emitted by the fragment. The dependence of $\bar{v}$ on mass and TKE can only be derived from the data once the $2 \mathrm{E}$-analysis is completed. As initial assumption we have 
used the evaluated data on $\bar{v}\left(m^{*}\right)$ from Wahl [11] and the parameterization

$$
\begin{aligned}
\bar{v}\left(m^{*}, \mathrm{TKE}\right) & =\bar{v}\left(m^{*}\right)+\frac{\bar{v}\left(m^{*}\right)}{\bar{v}\left(m^{*}\right)+\bar{v}\left(m_{c n}-m^{*}\right)} \cdot \Delta_{\mathrm{TKE}}, \\
\Delta_{\mathrm{TKE}} & =\frac{\overline{\operatorname{TKE}}\left(m^{*}\right)-\mathrm{TKE}}{E_{\mathrm{sep}}},
\end{aligned}
$$

where $E_{\text {sep }}=8.6 \mathrm{MeV} / \mathrm{n}$ is the average energy necessary to emit a neutron [12]. The analysis was later repeated using the results on $\bar{v}\left(m^{*}\right)$ and $E_{\text {sep }}=8.51 \mathrm{MeV} / \mathrm{n}$ derived from the data. No significant changes in the results were observed between the two analyses. In the 2E-technique the main contribution to the mass resolution is the neutron evaporation, since Eq. (2) only holds on average. In this work the mass resolution is 4-5 u (FWHM), determined by comparing the measured thermal mass yield to data from Ref. [13]. This mass resolution is close to what can be expected considering only the contribution from the neutron evaporation [14]. For events where neutrons and fission fragments are detected in coincidence, an additional correction [15] for the recoil energy imparted to the fragment is added to Eq. (2).

\section{Experimental results}

All results on correlations between fission fragment properties and PFN properties presented here are from the selected incident neutron energy range $[0.3 \mathrm{eV}, 45 \mathrm{keV}]$. Some of the results, presented in this section and originating from a subset of the data from the present experiment, has been reported upon earlier [16]. The main difference between results reported by us in Ref. [16] and reported here is, aside from the improved statistical accuracy, that the absolute neutron detection efficiency was re-evaluated. This effectively raised the mean total neutron multiplicity from 2.3 to 2.4 neutrons/fission.

\subsection{Prompt fission neutron angular distributions in the laboratory frame}

Most model calculations of the properties of PFNs are based on the assumption that the neutrons are emitted from the fragments after they have reached their terminal velocity. However, theoretical arguments have been raised asserting that at least a fraction of the neutrons are emitted during the scission process [17] and/or during the acceleration of the fragments [18, 19]. A large number of experiments have been devoted to investigate the source of prompt neutrons in detail. However, considering the variation of experimental results it is difficult to draw definitive conclusions. The experimental method used here, as well as in many earlier studies [20-22] of PFN emission in ${ }^{235} U(n, f)$, assumes that the neutrons are emitted from the fully accelerated fragment. Hence, it is of great importance to investigate to what extent the experimental data support this assumption. In order to do so, we follow a procedure similar to that of Vorobyev et al. [23]. The energy-angle distribution of neutrons emitted from moving fragments when observed in the laboratory frame is

$$
N\left(E_{n}, \theta\right)=N_{L}\left(\eta_{L}, \vartheta_{L}\right) \sqrt{\frac{E_{n}}{\eta_{L}}}+N_{H}\left(\eta_{H}, \vartheta_{H}\right) \sqrt{\frac{E_{n}}{\eta_{H}}},
$$

where $N_{L, H}\left(\eta_{L, H}, \vartheta_{L, H}\right)$ are the center-of-mass (c.m.) energy-angle distributions of neutrons from the light and heavy fragments, respectively. The neutron energies in the c.m. frame $\eta_{L, H}$ can be derived from experimental observables in the laboratory frame; light $(\mathrm{L})$ and heavy $(\mathrm{H})$ fragment energies 
$E_{L, H}$ and masses $m_{L, H}$, neutron energy $E_{n}$ and angle $\theta$ between the neutron's and the light fragment's directions of motion

$$
\begin{aligned}
& \eta_{L}=E_{n}+E_{L} \frac{m}{m_{L}}-2 \sqrt{E_{n} E_{L} \frac{m}{m_{L}}} \cos \theta, \\
& \eta_{H}=E_{n}+E_{H} \frac{m}{m_{H}}+2 \sqrt{E_{n} E_{H} \frac{m}{m_{H}}} \cos \theta,
\end{aligned}
$$

where $m$ is the mass of the neutron. For the purpose of the model a single fragmentation is used,

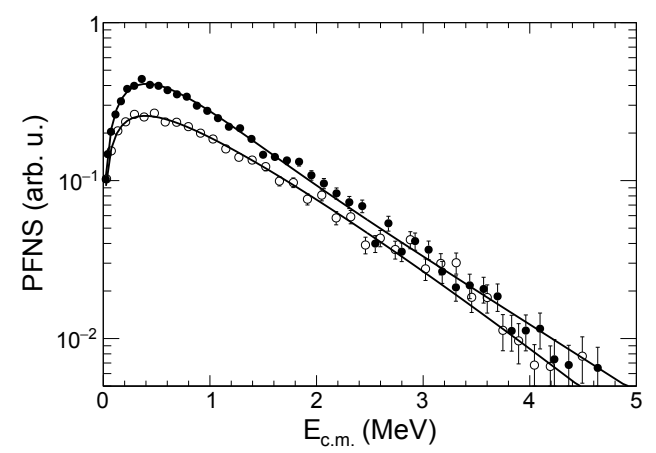

Figure 3. The c.m. energy spectra of prompt neutrons derived for the light $(\bullet)$ and heavy $(\circ)$ fragment groups.

with $E_{L} \frac{m}{m_{L}}=(1.02 \pm 0.01) \mathrm{MeV}$ and $E_{H} \frac{m}{m_{H}}=(0.491 \pm 0.01) \mathrm{MeV}$ taken as averages from the experimental data. Under the assumption of isotropic emission from the fully accelerated fragments, only the neutron spectra in the c.m. frame are unknown. They are determined from the experimental data by selecting small angles in the laboratory frame, where the contribution of neutrons from the complementary fragment is the smallest. Neutrons detected at angles smaller than $12^{\circ}$ relative to the motion of the light and heavy fission fragments are selected. The observed laboratory spectra for these selections are transformed into the c.m. frame of the respective fragment and corrected for the small contribution of neutrons from the complementary fragment, the result is displayed in Fig. 3. Each of the spectra has been fitted with a linear superposition of a Maxwellian and a Watt spectrum shape, represented by the full black lines. The fitted shapes are then used to calculate the spectrum as a function of the angle between the light fission fragment direction and the detected neutron, according to Eq. (4). The result of the calculation is presented as a contour plot in Fig. 4. As can be seen, the contour lines of the model do follow the contour lines of the experimental data quite well. In Fig. 5 the zeroth (A) and the first (B) moments of the calculated and experimentally observed spectra as a function of $\cos \theta$ are compared. The agreement is fair, although a slight underestimation of the experimental data is evident. The underestimation amounts to $2.5 \%$ of the total neutron yield. The description of the experimental data could be improved by assuming a small anisotropy in the c.m. frame [23]. However, we did not include an anisotropy term in the evaluation of the laboratory data as the angular distribution observed in the c.m. frame does not allow for this degree of freedom, see Sect. 4.2.3.

\subsection{Experimental results in the fission fragment rest-frame}

The complete experimental determination of all relevant kinematic parameters allows the transformation from the laboratory frame of reference into the rest frame of the fully accelerated fission fragments. This transformation is, however, complicated by the fact that for each laboratory angle only the 


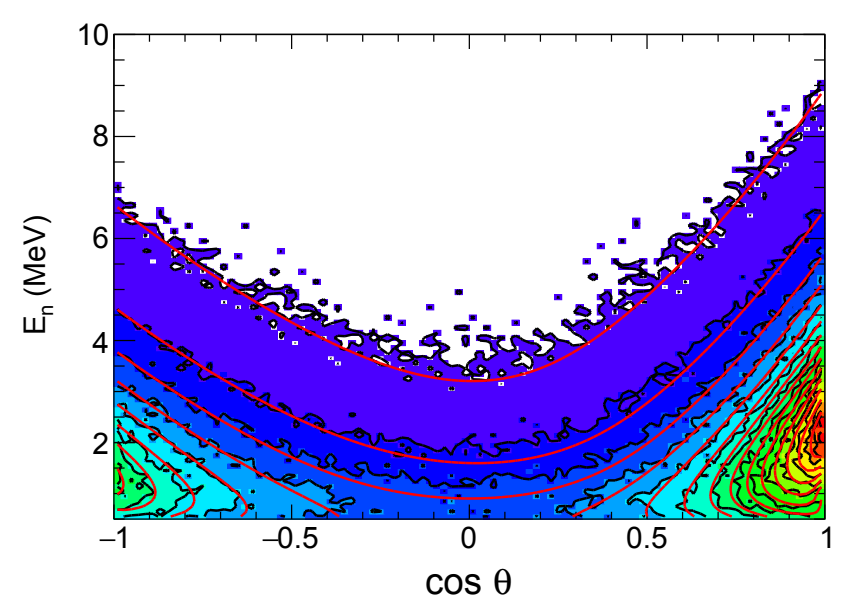

Figure 4. The laboratory energy-angle distribution of prompt neutrons. The experimental data are represented by a contour-plot with black contour lines on top of a color-coded scatter-plot. The red contour lines represent the model calculation according to Eq. (4) with adjusted parameters, see text for details.
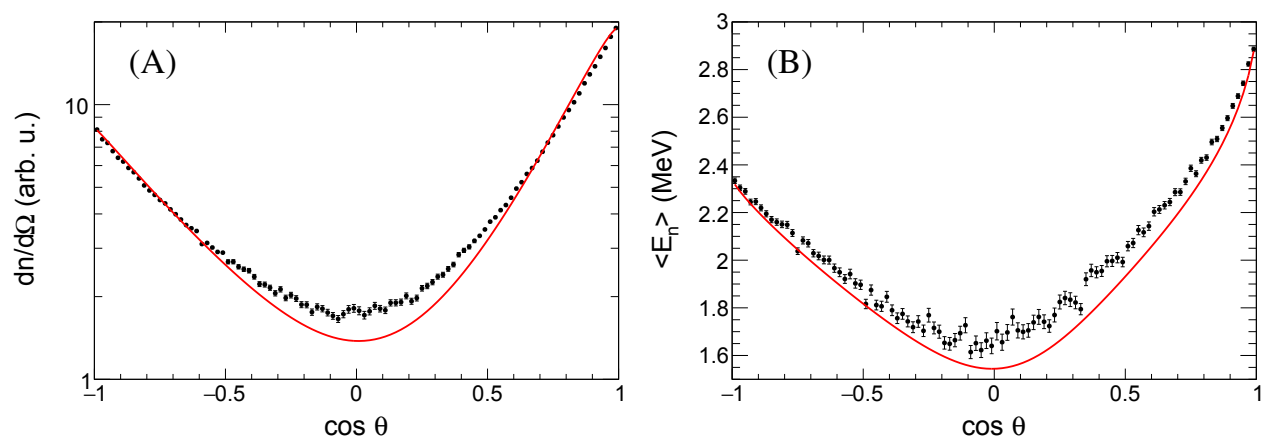

Figure 5. (A) Projection of the data in Fig. 4 onto $\cos \theta$. (B) The Average neutron energy as a function of $\cos \theta$. The red lines represent the result of the model calculation.

sum of contributions from the two fission fragments is observed. Thus, an unknown contribution must first be subtracted from any observed distribution. Fortunately, the contribution of neutrons emitted from the fragment flying away from the neutron detector is small. Therefore, in a first approximation it is assumed that all neutrons that are detected with a c.m. angle smaller than $90^{\circ}$ originate from the fragment directed towards the neutron detector. The resulting distribution is then used to calculate the disturbing component from the complementary fragment. The magnitude of the correction as a function of fission fragment mass is shown in Fig. 6.

\subsubsection{Angular distribution}

Using the procedures discussed above the angular distribution of prompt neutrons in the rest frame of the fully accelerated fragments has been determined. Figure 7 shows the angular distribution integrated over all c.m. neutron energies and all fission configuration, i.e. no selection in mass or TKE has been made. The result has been fitted with a second order Legendre polynomial. Obviously, the result supports isotropic emission in the c.m.-frame. This does, however, not exclude emission from non 


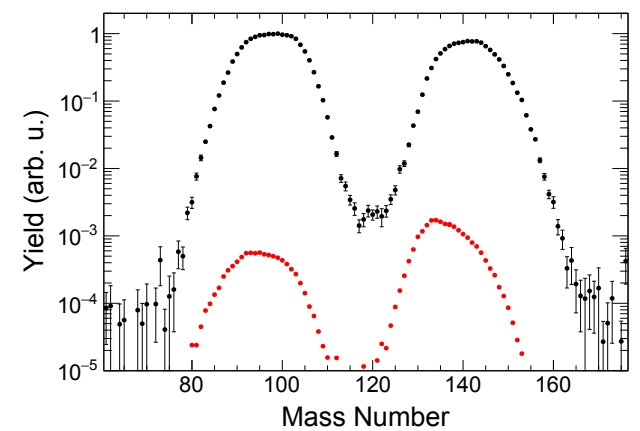

Figure 6. Fission neutron yield for $\theta_{\text {c.m. }}>90^{\circ}$ as a function of the fission fragment mass. The red points represent the calculated contribution of neutrons from the complementary fission fragment.

fully accelerated fragments due to the selection of events with $\theta_{\text {c.m. }}>90^{\circ}$. The selection implies that events with laboratory neutron energies smaller than the fragment energy per nucleon are not taken into account.

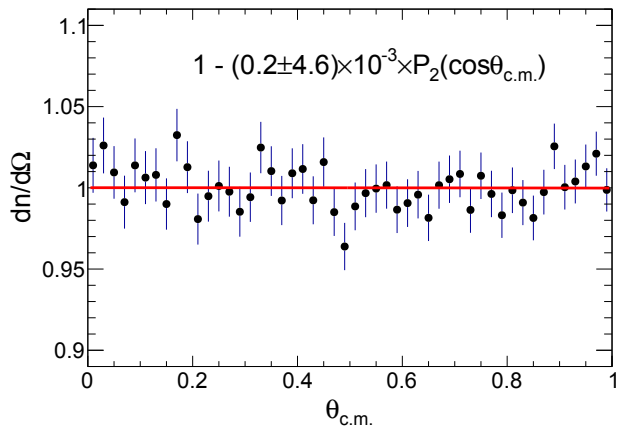

Figure 7. The angular distribution of prompt neutrons in the c.m.frame integrated over all neutron energies and fission configurations.

\subsubsection{Average neutron multiplicity}

The average neutron multiplicity as a function of the fragment mass is shown in Fig. 8 (A). For comparison, experimental data from Refs. [20, 21] and the evaluation from Ref. [11] are also shown in the figure. The general shape is reproduced in this work, however the minima around mass number $\sim 80 \mathrm{u}$ for the light fragments and $\sim 130 \mathrm{u}$ for the heavy fragments appear more pronounced in the present data. The average neutron multiplicity per fission is shown as a function of the heavy fragment mass in Fig. 8 (B). A pronounced minimum close to heavy fragment mass $A_{H} \sim 132 \mathrm{u}$ observed. The shape of the present data agrees very well with the data from Maslin et al. [22].

In Fig. 9 the average neutron multiplicity per fission is plotted as a function of the fragment TKE. As expected, a close to linear dependence is observed, except for TKE $\lesssim 145 \mathrm{MeV}$. A least square fit, indicated by the full black line in Fig. 9(A), results in $-\partial \mathrm{TKE} / \partial \bar{v}=12.0 \mathrm{MeV} / \mathrm{n}$. This value is substantially lower than the values $16.7-18.5 \mathrm{MeV} / \mathrm{n}$ determined in earlier studies, performed at thermal incident neutron energies [20-22, 24]. Data from Refs. [20-22, 24] are also included in Fig. 9(A). By studying the fission fragment TKE distributions from Refs. [20-22] it has been found that these studies suffer from substantial resolution broadening. The broadening also affects the correlation with detected neutrons, which explains the strong difference between the present data and 

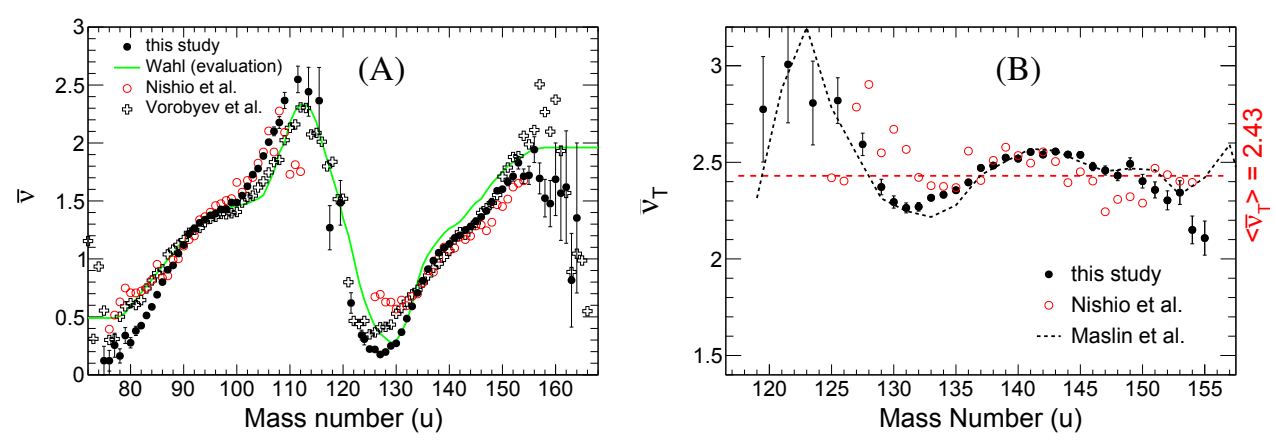

Figure 8. (A) The average neutron multiplicity per fragment as a function of the fragment mass from this study compared to data from Refs. [20,21] and the evaluation by Wahl [11]. (B) The average neutron multiplicity per fission as a function of the heavy fragment mass from this study compared to data from Refs. [20, 22]. The dashed red line indicates the mean multiplicity per fission.

the literature data. This conclusion was already drawn form a subset of the present data, and a more detailed discussion of this has been published in Ref. [16].
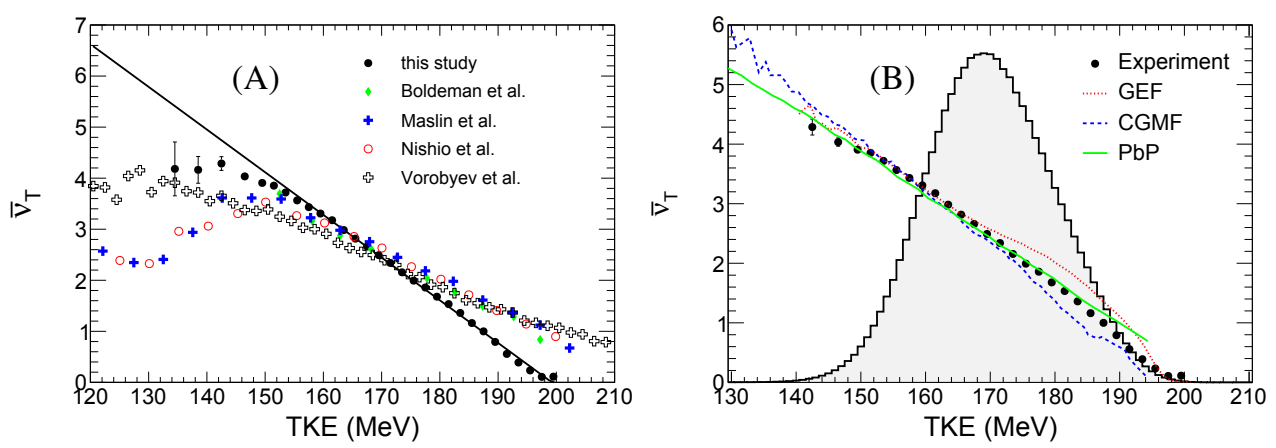

Figure 9. The average neutron multiplicity per fission as a function of the TKE. (A) The result from this study is compared to experimental data from Refs.[20-22, 24]. The black line represent a straight line with an inverse slope of $12.0 \mathrm{MeV} / \mathrm{n}$, obtained from a least square fit of the data from this study. (B) The result from this study is compared to model calculations $[1,25]$. The fission yield as a function of TKE determined in this study is also shown.

A comparison of the experimental result for average neutron multiplicity per fission $\bar{v}_{T}$ (TKE) with different model calculations, taken from a recent review of the available fission fragment deexcitation models [1, 25], is shown in Fig. 9(B). The different models presented in Ref. [1] agree well with each other. Therefore, not all of them are included in the figure. It is clear that the model calculations are in much better agreement with the present data set than with earlier experiment. 

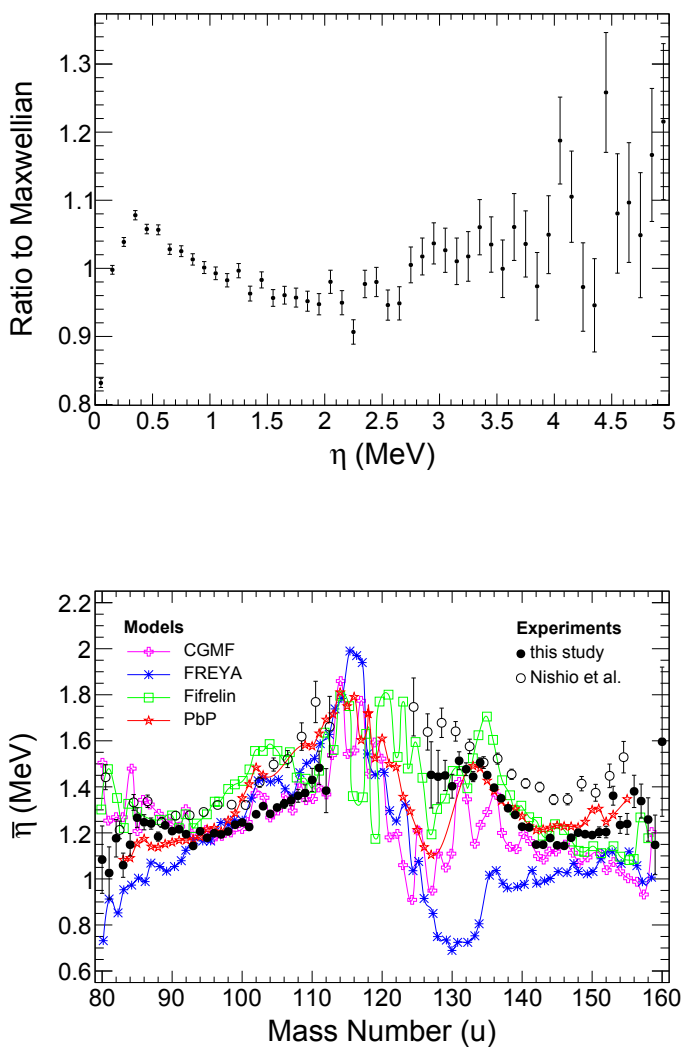

Figure 10. The prompt neutron spectrum in the c.m. frame, integrated over all fragmentations, presented as a ratio to a Maxwellian with temperature $0.826 \mathrm{MeV}$.

Figure 11. The average c.m. neutron energy $\bar{\eta}$ as a function of the fission fragment mass, results from this experiment are compared to model predictions from Refs. [1] and experimental data from Ref. [20].

\subsubsection{Prompt neutron energy}

The neutron energy in the c.m. frame $\eta$ has also been evaluated on an event-by-event basis. The integral spectrum is presented as a ratio to a Maxwellian with a fitted temperature of $0.826 \mathrm{MeV}$ in Fig. 10. The average neutron energy as a function of the fission fragment mass is compared to theoretical calculations and an earlier experiment in Fig. 11. The average values for the light- and heavy fragment groups are $1.23 \mathrm{MeV}$ and $1.26 \mathrm{MeV}$, respectively. The average c.m. neutron energy is an important validation parameter for neutron emission models as it relates to both the excitation energy partition between the fragments as well as the distribution of temperatures in the sequential emission of neutrons. The quantity is also important for application as it has a direct influence on the laboratory neutron spectrum. It is evident from Fig. 11 that $\bar{\eta}(A)$ is almost symmetric with respect to symmetric mass division, in contradiction to $\bar{v}(A)$, which shows the well know sawtooth shape. This is very similar to earlier findings for the prompt fission neutrons from ${ }^{252} \mathrm{Cf}(\mathrm{sf})$ [26-28]. The different model predictions are in general in good agreement with the experimental data. The FREYA model does however predict a pronounced sawtooth shape which is not observed in the experiment. All models predict a more or less pronounced minimum in the region below $\mathrm{A}=130$, while the experimental data shows only a flattening of the trend when approaching this region from heavier masses. Considering the strong slope of the mass yield curve and the experimental mass resolution it is expected that sharp changes in the properties of neutrons as a function of mass are washed out. 


\section{Conclusion}

A multi-parameter experiment on correlations between the properties of fission fragments and PFNs in the reaction ${ }^{235} \mathrm{U}(\mathrm{n}, \mathrm{f})$ for an incident neutron energy range $[0.3 \mathrm{eV}, 45 \mathrm{keV}]$ has been presented. The laboratory angular distribution of prompt neutrons have been compared with a model calculation based on isotropic emission from fully accelerated fragments. The model agrees fairly well with the experimental data, however the model calculation does underestimate the neutron yield at large angles with the fission axis. The underestimation amounts to $2.5 \%$ of the total neutron yield. Results on average neutron multiplicities in correlation with fission fragment mass and TKE show significant differences to earlier studies on this reaction, induced by thermal neutrons. The sawtooth shape of the average neutron multiplicity per fragment show more pronounced minima at $A \approx 130$ and $A \approx 80$. The TKE dependence of the neutron multiplicity per fission shows an inverse slope $\partial$ TKE / $\partial \bar{v}$ approximately $35 \%$ weaker than observed in earlier studies [20-22, 24]. The difference can be attributed to improved fission fragment TKE resolution in the present experiment. The present result for the average neutron multiplicity as a function of TKE is in good agreement with model calculations $[1,25]$.

\section{References}

[1] R. Capote, Y.-J. Chen, F.-J. Hambsch, N.V. Kornilov, J.P. Lestone, O. Litaize, B. Morillon, D. Neudecker, S. Oberstedt, T. Ohsawa, N. Otuka, V.G. Pronyaev, A. Saxena, O. Serot, O.A. Shcherbakov, N.-C. Shu, D.L. Smith, P. Talou, A. Trkov, A.C. Tudora, R. Vogt, A.S. Vorobyev, Nucl. Data Sheets 131, 1 (2016)

[2] S. Lemaire, P. Talou, T. Kawano, M. Chadwick, D. Madland, Phys. Rev. C 72 (2005), cited By 71

[3] N.V. Kornilov, F.-J. Hambsch, A.S. Vorobyev, Nucl. Phys. A 789, 55 (2007)

[4] A. Göök, W. Geerts, F.-J. Hambsch, S. Oberstedt, M. Vidali, S. Zeynalov, Nucl. Instr. and Meth. A 830, 366 (2016)

[5] N.V. Kornilov, Tech. rep., IAEA, INDC(USA)-108 (2015)

[6] N.V. Kornilov, I. Fabry, S. Oberstedt, F.-J. Hambsch, Nucl. Instr. and Meth. A 599, 226 (2009)

[7] V.V. Verbinski, W.R. Burrus, T.A. Love, W. Zobel, N.W. Hill, R. Textor, Nucl. Instr. and Meth. 65, 8 (1968)

[8] M. Tajik, N. Ghal-Eh, G.R. Etaati, H. Afarideh, Nucl. Instr. and Meth. A 704, 104 (2013)

[9] W. Mannhart, IAEA-TECDOC-410 (Leningrad, 1986), p. 158

[10] N.V. Kornilov, F.-J. Hambsch, I. Fabry, S. Oberstedt, T. Belgya, Z. Kis, L. Szentmiklosi, S. Simakov., Nucl. Sci. and Eng. 165, 117 (2010)

[11] C. Wahl, Atomic Data and Nuclear Data Tables 39, 56 (1988)

[12] H. Nifenecker, C. Signarbieux, R. Babinet, J. Poitou, 3rd IAEA Symp. on the Physics and Chemistry of Fission 2, 117 (1973)

[13] P. Geltenbort, F. Gönnenwein, A. Oed, Radiation Effects 93, 57 (1986)

[14] J. Terrell, Phys. Rev. 127, 880 (1962)

[15] A. Gavron, Nucl. Instr. and Meth. 115, 99 (1974)

[16] A. Göök, F.-J. Hambsch, S. Oberstedt, EPJ Web of Conf. ND 2016 Int. Conf. on Nucl. Data for Sci. and Tech. (2016)

[17] M. Rizea, N. Carjan, Nucl. Phys. A 909, 50 (2013)

[18] V.P. Eismont, Jour. Nucl. Energy A/B 20, 875 (1965)

[19] V.P. Eismont, Atomnya Energiya 19, 113 (1965) 
[20] K. Nishio, Y. Nakagome, H. Yamamoto, I. Kimura, Nucl. Phys. A 632, 540 (1998)

[21] A.S. Vorobyev, O. Shcherbakov, A. Gagarski, G. Val'ski, G. Petrov, EPJ Web of Conf. 8, 03004 (2010)

[22] E.E. Maslin, A.L. Rodgers, W.G.F. Core, Phys. Rev. 164, 1520 (1967)

[23] A.S. Vorobyev, O.A. Shcherbakov, Yu.S. Pleva, A.M Gagarski, G.V. Val'ski, G.A. Petrov, V.I. Petrova, T.A. Zavarukhina, Nucl. Instr. and Meth. A 598, 795 (2009)

[24] J.W. Boldeman, A.R. de L. Musgrove, R. L. Walsh, Aust. J. Phys. 24, 821 (1971)

[25] K.H. Schmidt, B. Jurado, C. Amouroux, C. Schmitt, Nucl. Data Sheets 131, 107 (2016)

[26] A. Göök, F.-J. Hambsch, M. Vidali, Phys. Rev. C 90, 064611 (2014)

[27] C. Budtz-Jørgensen, H.H. Knitter, Nucl. Phys. A 490, 307 (1988)

[28] H.R. Bowman, S.G. Thompson, J.C.D. Milton, W.J. Swiatecki, Phys. Rev. 126, 2120 (1962) 
